\title{
A CHARACTERIZATION OF THE $n$-ARY MANY-SORTED CLOSURE OPERATORS AND A MANY-SORTED TARSKI IRREDUNDANT BASIS THEOREM
}

\author{
J. CLIMENT VIDAL AND E. COSME LLÓPEZ
}

\begin{abstract}
A theorem of single-sorted algebra states that, for a closure space $(A, J)$ and a natural number $n$, the closure operator $J$ on the set $A$ is $n$-ary if, and only if, there exists a single-sorted signature $\Sigma$ and a $\Sigma$-algebra $\mathbf{A}$ such that every operation of $\mathbf{A}$ is of an arity $\leq n$ and $J=\operatorname{Sg}_{\mathbf{A}}$, where $\operatorname{Sg}_{\mathbf{A}}$ is the subalgebra generating operator on $A$ determined by $\mathbf{A}$. On the other hand, a theorem of Tarski asserts that if $J$ is an $n$-ary closure operator on a set $A$ with $n \geq 2$, and if $i<j$ with $i, j \in \operatorname{IrB}(A, J)$, where $\operatorname{IrB}(A, J)$ is the set of all natural numbers $n$ such that $(A, J)$ has an irredundant basis (三 minimal generating set) of $n$ elements, such that $\{i+1, \ldots, j-1\} \cap \operatorname{IrB}(A, J)=\varnothing$, then $j-i \leq n-1$. In this article we state and prove the many-sorted counterparts of the above theorems. But, we remark, regarding the first one under an additional condition: the uniformity of the many-sorted closure operator.
\end{abstract}

\section{Introduction.}

A well-known theorem of single-sorted algebra states that, for a closure space $(A, J)$ and a natural number $n \in \mathbb{N}=\omega$, the closure operator $J$ on the set $A$ is $n$-ary if, and only if, there exists a single-sorted signature $\Sigma$ and a $\Sigma$-algebra $\mathbf{A}$ such that every operation of $\mathbf{A}$ is of an arity $\leq n$ and $J=\operatorname{Sg}_{\mathbf{A}}$, where $\operatorname{Sg}_{\mathbf{A}}$ is the subalgebra generating operator on $A$ determined by $\mathbf{A}$. On the other hand, in [3], it was stated that, for an algebraic many-sorted closure operator $J$ on an $S$-sorted set $A, J=\operatorname{Sg}_{\mathbf{A}}$ for some many-sorted signature $\Sigma$ and some $\Sigma$-algebra A if, and only if, $J$ is uniform. And, by using, among others, the just mentioned result, our first main result is the following characterization of the $n$-ary many-sorted closure operators: Let $S$ be a set of sorts, $A$ an $S$-sorted set, $J$ a many-sorted closure operator on $A$, and $n \in \mathbb{N}$. Then $J$ is $n$-ary and uniform if, and only if, there exists an $S$-sorted

Date: May 5th, 2016.

2010 Mathematics Subject Classification. Primary: 06A15; Secondary: 54A05.

Key words and phrases. $S$-sorted set, delta of Kronecker, support of an $S$-sorted set, $n$-ary many-sorted closure operator, uniform many-sorted closure operator, irredundant basis for a many-sorted closure space. 
signature $\Sigma$ and a $\Sigma$-algebra $\mathbf{A}$ such that $J=\operatorname{Sg}_{\mathbf{A}}$ and every operation of $\mathbf{A}$ is of an arity $\leq n$.

We turn next to Tarski's irredundant basis theorem for single-sorted closure spaces. But before doing that let us begin by recalling the terminology relevant to the case. Given an $n$ in $\mathbb{N}$, a set $A$, and a closure operator $J$ on $A$, the closure operator $J$ is said to be an $n$-ary closure operator on $A$ if $J=J_{\leq n}^{\omega}$, where $J_{\leq n}^{\omega}$ is the supremum of the family $\left(J_{<n}^{m}\right)_{m \in \omega}$ of operators on $A$ defined by recursion as follows: for $m=0, J_{\leq n}^{0}=\operatorname{Id}_{\operatorname{Sub}(A)}$; for $m=k+1$, with $k \geq 0, J_{\leq n}^{k+1}(X)=J_{\leq n} \circ J_{\leq n}^{k}$, where $J_{\leq n}$ is the operator on $A$ defined, for every $X \subseteq A$, as follows:

$$
J_{\leq n}(X)=\bigcup\left\{J(Y) \mid Y \in \operatorname{Sub}_{\leq n}(X)\right\},
$$

where $\operatorname{Sub}_{\leq n}(X)$ is $\{Y \subseteq X \mid \operatorname{card}(Y) \leq n\}$.

Alfred Tarski in [4, on pp. 190-191, proved, as reformulated by S. Burris and H. P. Sankappanavar in [2], on pp. 33-34, the following theorem. Given a set $A$ and an $n$-ary closure operator $J$ on $A$ with $n \geq 2$, for every $i, j \in \operatorname{IrB}(A, J)$, where $\operatorname{IrB}(A, J)$ is the set of all natural numbers $n$ such that $(A, J)$ has an irredundant basis ( $\equiv$ minimal generating set) of $n$ elements, if $i<j$ and $\{i+1, \ldots, j-1\} \cap \operatorname{IrB}(A, J)=$ $\varnothing$, then $j-i \leq n-1$. Thus, as stated by Burris and Sankappanavar in [2], on p. 33, the length of the finite gaps in $\operatorname{IrB}(A, J)$ is bounded by $n-2$ if $J$ is an $n$-ary closure operator. And our second main result is the proof of Tarski's irredundant basis theorem for many-sorted closure spaces.

\section{MANY-SORTED SETS, MANY-SORTED ClOSURE OPERATORS, AND MANY-SORTED ALGEBRAS.}

In this section, for a set of sorts $S$ in a fixed Grothendieck universe $\mathcal{U}$, we begin by recalling some basic notions of the theory of $S$-sorted sets, e.g., those of subset of an $S$-sorted set, of proper subset of an $S$-sorted set, of delta of Kronecker, of cardinal of an $S$-sorted set, and of support of an S-sorted set; and by defining, for an $S$-sorted set $A$, the concepts of many-sorted closure operator on $A$ and of many-sorted closure space. Moreover, for a many-sorted closure operator $J$ on $A$, we define the notions of irredundant or independent part of $A$ with respect to $J$, of basis or generator of $A$ with respect to $J$, of irredundant basis of $A$ with respect to $J$, and of minimal basis of $A$ with respect to $J$. In addition, we state that the notion of irredundant basis of $A$ with respect to $J$ is equivalent to the notion of minimal basis of $A$ with respect to $J$ and, afterwards, for a many-sorted closure space $(A, J)$, we define the subset $\operatorname{IrB}(A, J)$ of $\mathbb{N}$ as being formed by choosing those natural numbers which are the cardinal of an irredundant basis of $A$ with respect to $J$. On the other hand, for a natural number $n$, we define the concept of $n$-ary many-sorted closure operator on $A$ and provide a 
characterization of the $n$-ary many-sorted closure operators $J$ on $A$, in terms of the fixed points of $J$. Besides, for a set of sorts $S$, we define the concept of $S$-sorted signature, and, for an $S$-sorted signature $\Sigma$, the notion of $\Sigma$-algebra and, for a $\Sigma$-algebra $\mathbf{A}$, the concept of subalgebra of $\mathbf{A}$ and the subalgebra generating many-sorted operator $\mathrm{Sg}_{\mathbf{A}}$ on $A$ determined by A. Subsequently, once defined the notion of finitely generated $\Sigma$-algebra, we state that, for a finitely generated $\Sigma$-algebra $\mathbf{A}, \operatorname{IrB}\left(A, \operatorname{Sg}_{\mathbf{A}}\right) \neq \varnothing$.

Definition 2.1. An $S$-sorted set is a function $A=\left(A_{s}\right)_{s \in S}$ from $S$ to $\mathcal{U}$.

Definition 2.2. Let $S$ be a set of sorts. If $A$ and $B$ are $S$-sorted sets, then we will say that $A$ is a subset of $B$, denoted by $A \subseteq B$, if, for every $s \in S, A_{s} \subseteq B_{s}$, and that $A$ is a proper subset of $B$, denoted by $A \subset B$, if $A \subseteq B$ and, for some $s \in S, B_{s}-A_{s} \neq \varnothing$. We denote by $\operatorname{Sub}(A)$ the set of all $S$-sorted sets $X$ such that $X \subseteq A$.

Definition 2.3. Given a sort $t \in S$ and a set $X$ we call delta of Kronecker for $(t, X)$ the $S$-sorted set $\delta^{t, X}$ defined, for every $s \in S$, as follows:

$$
\delta_{s}^{t, X}= \begin{cases}X, & \text { if } s=t ; \\ \varnothing, & \text { otherwise. }\end{cases}
$$

For a final set $\{x\}$, to abbreviate, we will write $\delta^{t, x}$ instead of the more accurate $\delta^{t,\{x\}}$.

We next define, for a set of sorts $S$, the concept of cardinal of an $S$-sorted set, for an $S$-sorted set $A$, the notion of support of $A$, and characterize the finite $S$-sorted sets in terms of its supports.

Definition 2.4. Let $A$ be an $S$-sorted set. Then the cardinal of $A$, denoted by $\operatorname{card}(A)$, is the cardinal of $\coprod A$, where $\coprod A$, the coproduct of $A=\left(A_{s}\right)_{s \in S}$, is $\bigcup_{s \in S}\left(A_{s} \times\{s\}\right)$. Moreover, $\operatorname{Sub}_{\text {fin }}(A)$ denotes the set of all finite subsets of $A$, i.e., the set $\left\{X \subseteq A \mid \operatorname{card}(X)<\aleph_{0}\right\}$, and, for a natural number $n, \operatorname{Sub}_{\leq n}(A)$ denotes the set of all subsets of $A$ with at most $n$ elements, i.e., the set $\{X \subseteq A \mid \operatorname{card}(X) \leq n\}$. Sometimes, for simplicity of notation, we write $X \subseteq$ fin $A$ instead of $X \in \operatorname{Sub}_{\text {fin }}(A)$.

Definition 2.5. Let $S$ be a set of sorts. Then the support of $A$, denoted by $\operatorname{supp}_{S}(A)$, is the set $\left\{s \in S \mid A_{s} \neq \varnothing\right\}$.

Proposition 2.6. An $S$-sorted set $A$ is finite if, and only if, $\operatorname{supp}_{S}(A)$ is finite and, for every $s \in \operatorname{supp}_{S}(A), \operatorname{card}\left(A_{s}\right)<\aleph_{0}$.

Definition 2.7. Let $S$ be a set of sorts and $A$ an $S$-sorted set. A many-sorted closure operator on $A$ is a mapping $J$ from $\operatorname{Sub}(A)$ to $\operatorname{Sub}(A)$, which assigns to every $X \subseteq A$ its $J$-closure $J(X)$, such that, for every $X, Y \subseteq A$, satisfies the following conditions:

(1) $X \subseteq J(X)$, i.e., $J$ is extensive. 
(2) If $X \subseteq Y$, then $J(X) \subseteq J(Y)$, i.e., $J$ is isotone.

(3) $J(J(X))=J(X)$, i.e., $J$ is idempotent.

Given two many-sorted closure operators $J$ and $K$ on $A, J$ is called smaller than $K$, denoted by $J \leq K$, if, for every $X \subseteq A, J(A) \subseteq K(A)$. A many-sorted closure space is an ordered pair $(A, J)$ where $A$ is an $S$-sorted set and $J$ a many-sorted closure operator on $A$. Moreover, if $X \subseteq A$, then $X$ is irredundant (or independent) with respect to $J$ if, for every $s \in S$ and every $x \in X_{s}, x \notin J\left(X-\delta^{s, x}\right)_{s}, X$ is a basis (or a generator) with respect to $J$ if $J(X)=A, X$ is an irredundant basis with respect to $J$ if $X$ irreduntant and a basis with respect to $J$, and $X$ is a minimal basis with respect to $J$ if $J(X)=A$ and, for every $Y \subset X$, $J(Y) \neq A$.

We next state that the notion of irredundant basis of $A$ with respect to $J$ is equivalent to the notion of minimal basis of $A$ with respect to $J$. Moreover, for a many-sorted closure space $(A, J)$, we define $\operatorname{IrB}(A, J)$ as the intersection of the set of all natural numbers and the set of the cardinals of the irredundant basis of $A$ with respect to $J$.

Proposition 2.8. Let $(A, J)$ be a many-sorted closure space and $X \subseteq A$. Then $X$ is an irredundant basis with respect to $J$ if, and only if, it is a minimal basis with respect to $J$.

Definition 2.9. Let $S$ be a set of sorts and $(A, J)$ a many-sorted closure space. Then we denote by $\operatorname{IrB}(A, J)$ the subset of $\mathbb{N}$ defined as follows:

$$
\operatorname{IrB}(A, J)=\mathbb{N} \cap\left\{\operatorname{card}(X) \mid \begin{array}{c}
X \text { is an irredundant basis } \\
\text { of } A \text { with respect to } J
\end{array}\right\} .
$$

Later, in this section, after having defined, for a set of sorts $S$ and an $S$-sorted signature $\Sigma$, the concept of $\Sigma$-algebra, for a $\Sigma$-algebra $\mathbf{A}=(A, F)$, the uniform algebraic many-sorted closure operator $\mathrm{Sg}_{\mathbf{A}}$ on $A$, called the subalgebra generating many-sorted operator on $A$ determined by $\mathbf{A}$, and the notion of finitely generated $\Sigma$-algebra, we will state that, for a finitely generated $\Sigma$-algebra $\mathbf{A}, \operatorname{IrB}\left(A, \operatorname{Sg}_{\mathbf{A}}\right) \neq \varnothing$.

Definition 2.10. Let $A$ be an $S$-sorted set, $J$ a many-sorted closure operator on $A$, and $n$ a natural number.

(1) We denote by $J_{\leq n}$ the many-sorted operator on $A$ defined, for every $X \subseteq A$, as follows:

$$
J_{\leq n}(X)=\bigcup\left\{J(Y) \mid Y \in \operatorname{Sub}_{\leq n}(X)\right\} .
$$

(2) We define the family $\left(J_{\leq n}^{m}\right)_{m \in \mathbb{N}}$ of many-sorted operator on $A$, recursively, as follows:

$$
J_{\leq n}^{m}= \begin{cases}\operatorname{Id}_{\operatorname{Sub}(A)}, & \text { if } m=0 ; \\ J_{\leq n} \circ J_{\leq n}^{k}, & \text { if } m=k+1, \text { with } k \geq 0 .\end{cases}
$$


(3) We denote by $J_{\leq n}^{\omega}$ the many-sorted operator on $A$ that assigns to an $S$-sorted subset $X$ of $A, J_{\leq n}^{\omega}(X)=\bigcup_{m \in \mathbb{N}} J_{\leq n}^{m}(X)$.

(4) We say that $J$ is $n$-ary if $J=J_{\leq n}^{\underline{\omega}}$.

Remark. Let $J$ be a many-sorted closure operator on $A$. Then $J$ is 0-ary, i.e., $J=J_{\leq 0}^{\omega}$, if, and only if, for every $X \subseteq A$, we have that

$$
J(X)=X \cup J\left(\varnothing^{S}\right),
$$

where $\varnothing^{S}$ is the $S$-sorted set whose $s$ th coordinate, for every $s \in S$, is $\varnothing$.

We next prove that $J$ is 1 -ary, i.e., that $J=J_{\leq 1}^{\omega}$, if and only if, for every $X \subseteq A$, we have that

$$
J(X)=J\left(\varnothing^{S}\right) \cup \bigcup_{s \in S, x \in X_{s}} J\left(\delta^{s, x}\right) .
$$

Let us suppose that, for every $X \subseteq A, J(X)=J\left(\varnothing^{S}\right) \cup \bigcup_{s \in S, x \in X_{s}} J\left(\delta^{s, x}\right)$. Then it is obvious that, for every $X \subseteq A, J(X) \subseteq J_{<1}(X)$. Let us verify that, for every $X \subseteq A, J_{\leq 1}(X)=\bigcup\left\{J(Y) \mid Y \in \operatorname{Sub}_{\leq 1}(X)\right\} \subseteq J(X)$. Let $Y$ be an element of $\operatorname{Sub}_{<1}(X)$. Then $Y=\varnothing^{S}$ or $Y=\delta^{t, a}$, for some $t \in S$ and some $a \in X_{t}$. If $Y=\varnothing^{S}$, then

$$
J\left(\varnothing^{S}\right) \subseteq J\left(\varnothing^{S}\right) \cup \bigcup_{s \in S, x \in X_{s}} J\left(\delta^{s, x}\right)=J(X) .
$$

If $Y=\delta^{t, a}$, then $J\left(\delta^{t, a}\right) \subseteq \bigcup_{s \in S, x \in X_{s}} J\left(\delta^{s, x}\right)$, hence

$$
J\left(\delta^{t, a}\right) \subseteq J\left(\varnothing^{S}\right) \cup \bigcup_{s \in S, x \in X_{s}} J\left(\delta^{s, x}\right)=J(X) .
$$

Thus $J_{\leq 1}(X) \subseteq J\left(\varnothing^{S}\right) \cup \bigcup_{s \in S, x \in X_{s}} J\left(\delta^{s, x}\right)=J(X)$. Therefore $J=J_{\leq 1}$. Hence, for every $m \geq 1, J=J_{<1}^{m}$. Consequently $J$ is 1-ary.

Reciprocally, let us suppose that $J$ is 1-ary, i.e., that, for every $X \subseteq$ $A, J(X)=\bigcup_{m \in \mathbb{N}} J_{\leq 1}^{m}(X)$. Then, obviously, we have that

$$
J(X) \supseteq J\left(\varnothing^{S}\right) \cup \bigcup_{s \in S, x \in X_{s}} J\left(\delta^{s, x}\right) .
$$

Let us verify that, for every $m \in \mathbb{N}, J\left(\varnothing^{S}\right) \cup \bigcup_{s \in S, x \in X_{s}} J\left(\delta^{s, x}\right) \supseteq J_{<1}^{m}$. Evidently $J\left(\varnothing^{S}\right) \cup \bigcup_{s \in S, x \in X_{s}} J\left(\delta^{s, x}\right) \supseteq J_{\leq 1}^{0}(X) \cup J_{\leq 1}^{1}(X)$. Let $k$ be $\geq 1$ and let us suppose that $J\left(\varnothing^{S}\right) \cup \bigcup_{s \in S, x \in X_{s}} J\left(\delta^{s, x}\right) \supseteq J_{\leq 1}^{k}(X)$. We will show that $J\left(\varnothing^{S}\right) \cup \bigcup_{s \in S, x \in X_{s}} J\left(\delta^{s, x}\right) \supseteq J_{\leq 1}^{k+1}(X)$. By definition we have that

$$
J_{\leq 1}^{k+1}(X)=J_{\leq 1}\left(J_{\leq 1}^{k}(X)\right)=\bigcup\left\{J(Z) \mid Z \in \operatorname{Sub}_{\leq 1}\left(J_{\leq 1}^{k}(X)\right)\right\} .
$$

Let $Z$ be an element of $\operatorname{Sub}_{\leq 1}\left(J_{\leq 1}^{k}(X)\right)$. Then $Z \subseteq J_{\leq 1}^{k}(X)$. But we have that $J_{\leq 1}^{k}(X)=\bigcup\left\{J(Y) \mid Y \in \operatorname{Sub}_{\leq 1}\left(J_{\leq 1}^{k-1}(X)\right)\right\}$. Therefore, for some $Y \in \operatorname{Sub}_{\leq 1}\left(J_{\leq 1}^{k-1}(X)\right), Z \subseteq J(Y)$. Thus $J(Z) \subseteq J(J(Y))=J(Y)$. But $J(Y) \subseteq J_{\leq 1}^{k}(X)$. Consequently $J(Z) \subseteq J_{\leq 1}^{k}(X)$. Whence, by the induction hypothesis, $J(Z) \subseteq J\left(\varnothing^{S}\right) \cup \bigcup_{s \in S, x \in X_{s}} J\left(\delta^{s, x}\right)$. From this, since $Z$ was an arbitrary element of $\operatorname{Sub}_{\leq 1}\left(J_{\leq 1}^{k}(X)\right)$, we infer that $J_{\leq 1}^{k+1}(X)=\bigcup\left\{J(Z) \mid Z \in \operatorname{Sub}_{\leq 1}\left(J_{\leq 1}^{k}(X)\right)\right\} \subseteq J\left(\varnothing^{S}\right) \cup \bigcup_{s \in S, x \in X_{s}} J\left(\delta^{s, x}\right)$. 
Thus, for every $X \subseteq A$, we have that

$$
J(X)=J\left(\varnothing^{S}\right) \cup \bigcup_{s \in S, x \in X_{s}} J\left(\delta^{s, x}\right) .
$$

Remark. Let $n$ be $\geq 1, A$ an $S$-sorted set, $X \subseteq A$, and $J$ a manysorted closure operator on $A$. Then, for every $k \geq 0$ and every $Y \subseteq A$, if $Y \in \operatorname{Sub}_{\leq n}\left(J_{\leq n}^{k}(X)\right)$, then $Y \in \operatorname{Sub}_{\leq n}\left(J_{\leq n}^{k+1}(X)\right)$.

We next state, for a natural number $n \geq 1$ and a many-sorted closure operator $J$ on an $S$-sorted set $A$, that the family of many-sorted operators $\left(J_{\leq n}^{m}\right)_{m \in \mathbb{N}}$ on $A$ is an ascending chain and that $J_{\leq n}^{\omega}$, which is the supremum of the above family, is the greatest $n$-ary many-sorted closure operator on $A$ which is smaller than $J$.

Proposition 2.11. For a natural number $n \geq 1$, an $S$-sorted set $A$, and a many-sorted closure operator $J$ on $A$, the family of many-sorted operators $\left(J_{\leq n}^{m}\right)_{m \in \mathbb{N}}$ on $A$ is an ascending chain, i.e., for every $m \in \mathbb{N}$, $J_{\leq n}^{m} \leq J_{\leq n}^{m+1}$. Moreover, $J_{\leq n}^{\omega}$ is the greatest $n$-ary many-sorted closure operator on $A$ such that $J_{\leq n}^{\underline{\omega}} \leq J$.

We next provide a characterization of the $n$-ary many-sorted closure operators $J$ on an $S$-sorted set $A$ in terms of the fixed points $X$ of $J$ and of its relationships with the $J$-closures of the subsets of $X$ with at most $n$ elements.

Proposition 2.12. Let $A$ be an $S$-sorted set, $J$ a many-sorted closure operator on $A$, and $n$ a natural number. Then $J$ is $n$-ary if, and only if, for every $X \subseteq A$, if, for every $Z \in \operatorname{Sub}_{\leq n}(X), J(Z) \subseteq X$, then $J(X)=X$ (i.e., if, and only if, for every $X \subseteq A, X$ is a fixed point of $J$ if $X$ contains the $J$-closure of each of its subsets with at most $n$ elements).

Proof. If $n=0$, then the result is obvious. So let us consider the case when $n \geq 1$. Let us suppose that $J$ is $n$-ary and let $X$ be a subset of $A$ such that, for every $Z \in \operatorname{Sub}_{\leq n}(X), J(Z) \subseteq X$. We want to show that $J(X)=X$. Because $J$ is extensive, $X \subseteq J(X)$. Therefore it only remains to show that $J(X) \subseteq X$. Since, by hypothesis, $J(X)=$ $\bigcup_{m \in \mathbb{N}} J_{\leq n}^{m}(X)$, to show that $J(X) \subseteq X$ it suffices to prove that, for every $m \in \mathbb{N}, J_{\leq n}^{m}(X) \subseteq X$.

For $m=0$ we have that $J_{\leq n}^{0}(X)=X \subseteq X$.

Let us suppose that, for $k \geq 0, J_{\leq n}^{k}(X) \subseteq X$. Then we want to show that $J_{\leq n}^{k+1}(X) \subseteq X$. But, by definition, we have that

$$
J_{\leq n}^{k+1}(X)=J_{\leq n}\left(J_{\leq n}^{k}(X)\right)=\bigcup\left\{J(Y) \mid Y \in \operatorname{Sub}_{\leq n}\left(J_{\leq n}^{k}(X)\right)\right\} .
$$

Hence what we have to prove is that, for every $Y \in \operatorname{Sub}_{\leq n}\left(J_{\leq n}^{k}(X)\right)$, $J(Y) \subseteq X$. Let $Y$ be a subset of $J_{\leq n}^{k}(X)$ such that $\operatorname{card}(Y) \leq n$. Since $J_{<n}^{k}(X) \subseteq X$, we have that $Y \subseteq X$ and $\operatorname{card}(Y) \leq n$, therefore $J(Y) \subseteq$ $X$. Consequently, for every $X \subseteq A$, if, for every $Z \in \operatorname{Sub}_{\leq n}(X)$, $J(Z) \subseteq X$, then $J(X)=X$. 
Reciprocally, let us suppose that, for every $X \subseteq A$, if, for every $Z \in \operatorname{Sub}_{\leq n}(X), J(Z) \subseteq X$, then $J(X)=X$. We want to show that $J$ is $n$-ary, i.e., that $J=J_{\leq n}^{\omega}$. Let $X$ a subset of $A$. Then it is obvious that $J_{\leq n}^{\omega}(X)=\bigcup_{m \in \mathbb{N}} J_{\leq n}^{m}(X) \subseteq J(X)$. We now proceed to prove that $J(X) \subseteq J_{\leq n}^{\omega}(X)$. Since $J$ is isotone and, by the definition of $J_{\leq n}^{\omega}$, $X \subseteq J_{\leq n}^{\omega}(\bar{X})$, we have that $J(X) \subseteq J\left(J_{\leq n}^{\omega}(X)\right)$. Therefore to prove that $J(X) \subseteq J_{\leq n}^{\omega}(X)$ it suffices to prove that $J\left(J_{\leq n}^{\omega}(X)\right)=J_{\leq n}^{\omega}(X)$. But the just stated equation follows from the supposition because, as we will prove next, for every $Z \in \operatorname{Sub}_{\leq n}\left(J_{\leq n}^{\omega}(X)\right)$, we have that $J(Z) \subseteq$ $J_{\leq n}^{\omega}(X)$. Let $Z$ be a subset of $J_{\leq n}^{\omega}(X)$ such that $\operatorname{card}(Z) \leq n$. Then,

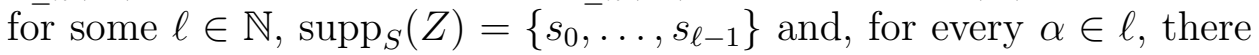
exists an $n_{\alpha} \in \mathbb{N}-1$ such that $Z_{s_{\alpha}}=\left\{z_{\alpha, 0}, \ldots, z_{\alpha, n_{\alpha}-1}\right\}$. Therefore, for every $\alpha \in \ell$ and every $\beta \in n_{\alpha}$ there exists an $m_{\alpha, \beta} \in \mathbb{N}$ such that that $z_{\alpha, \beta} \in J_{<n}^{m_{\alpha, \beta}}(X)_{s_{\alpha}}$. Since it may be helpful for the sake of understanding, let us represent the situation just described by the following figure:

$$
\begin{array}{ccc}
z_{0,0} \in J_{\leq n}^{m_{0,0}}(X)_{s_{0}} & \ldots & z_{0, n_{0}-1} \in J_{\leq n}^{m_{0, n_{0}-1}}(X)_{s_{0}} \\
\vdots & \ddots & \vdots \\
z_{\ell-1,0} \in J_{\leq n}^{m_{\ell-1,0}}(X)_{s_{\ell-1}} & \ldots & z_{\ell-1, n_{\ell-1}-1} \in J_{\leq n}^{m_{\ell-1, n_{\ell-1}-1}}(X)_{s_{\ell-1}}
\end{array}
$$

Hence, for every $\alpha \in \ell$ there exists a $\beta_{\alpha} \in n_{\alpha}$ such that $Z_{s_{\alpha}} \subseteq$ $J_{\leq n}^{m_{\alpha, \beta_{\alpha}}}(X)_{s_{\alpha}}$. On the other hand, since the family of many-sorted operators $\left(J_{\leq n}^{m}\right)_{m \in \mathbb{N}}$ on $A$ is an ascending chain, there exists an $m$ in the set $\left\{m_{\alpha, \beta_{\alpha}} \mid \alpha \in \ell\right\}$ such that, for every $\alpha \in \ell, J_{\leq n}^{m_{\alpha, \beta_{\alpha}}} \leq J_{\leq n}^{m}$. Thus $Z \subseteq J_{\leq n}^{m}(X)$. Therefore, since, in addition, $\operatorname{card}(Z) \leq n$, we have that $Z \in \operatorname{Sub}_{\leq n}\left(J_{\leq n}^{m}(X)\right)$. Thus

$$
J(Z) \subseteq J_{\leq n}^{m+1}(X)=J_{\leq n}\left(J_{\leq n}^{m}(X)\right)=\bigcup\left\{J(K) \mid K \in \operatorname{Sub}_{\leq n}\left(J_{\leq n}^{m}(X)\right)\right\} .
$$

Consequently $J(Z) \subseteq J_{\leq n}^{\omega}(X)$. Hence $J(X) \subseteq J_{\leq n}^{\omega}(X)$. Whence $J=$ $J_{\leq n}^{\omega}$, which completes the proof.

We next recall the notion of free monoid on a set and, for a set of sorts $S$, we define, by using the the just mentioned notion, the concept of $S$-sorted signature and, for an $S$-sorted signature $\Sigma$, the concept of $\Sigma$-algebra.

Definition 2.13. Let $S$ be a set of sorts. The free monoid on $S$, denoted by $\mathbf{S}^{\star}$, is $\left(S^{\star}, \curlywedge, \lambda\right)$, where $S^{\star}$, the set of all words on $S$, is $\bigcup_{n \in \mathbb{N}} \operatorname{Hom}(n, S)$, the set of all mappings $w: n \longrightarrow S$ from some $n \in \mathbb{N}$ to $S$, $\curlywedge$, the concatenation of words on $S$, is the binary operation on $S^{\star}$ which sends a pair of words $(w, v)$ on $S$ to the mapping $w \curlywedge v$ from $|w|+|v|$ to $S$, where $|w|$ and $|v|$ are the lengths (三 domains) of the 
mappings $w$ and $v$, respectively, defined as follows:

$$
w \curlywedge v\left\{\begin{aligned}
|w|+|v| & \longrightarrow S \\
i & \longmapsto \begin{cases}w_{i}, & \text { if } 0 \leq i<|w| ; \\
v_{i-|w|}, & \text { if }|w| \leq i<|w|+|v|,\end{cases}
\end{aligned}\right.
$$

and $\lambda$, the empty word on $S$, is the unique mapping $\lambda: \varnothing \longrightarrow S$.

Definition 2.14. Let $S$ be a set of sorts. Then an $S$-sorted signature is a function $\Sigma$ from $S^{\star} \times S$ to $\mathcal{U}$ which sends a pair $(w, s) \in S^{\star} \times S$ to the set $\Sigma_{w, s}$ of the formal operations of arity $w$, sort (or coarity) $s$, and rank (or biarity) $(w, s)$.

Definition 2.15. Let $\Sigma$ be an $S$-sorted signature and $A$ an $S$-sorted set. The $S^{\star} \times S$-sorted set of the finitary operations on $A$ is the family $\left(\operatorname{Hom}\left(A_{w}, A_{s}\right)\right)_{(w, s) \in S^{\star} \times S}$, where, for every $w \in S^{\star}, A_{w}=\prod_{i \in|w|} A_{w_{i}}$. A structure of $\Sigma$-algebra on $A$ is an $S^{\star} \times S$-mapping $F=\left(F_{w, s}\right)_{(w, s) \in S^{\star} \times S}$ from $\Sigma$ to $\left(\operatorname{Hom}\left(A_{w}, A_{s}\right)\right)_{(w, s) \in S^{\star} \times S}$. For a pair $(w, s) \in S^{\star} \times S$ and a formal operation $\sigma \in \Sigma_{w, s}$, in order to simplify the notation, the operation from $A_{w}$ to $A_{s}$ corresponding to $\sigma$ under $F_{w, s}$ will be written as $F_{\sigma}$ instead of $F_{w, s}(\sigma)$. A $\Sigma$-algebra is a pair $(A, F)$, abbreviated to A, where $A$ is an $S$-sorted set and $F$ a structure of $\Sigma$-algebra on $A$.

Since it will be used afterwards, we next define, for a set of sorts $S$ and an $S$-sorted set $A$, the notions of algebraic and of uniform manysorted closure operator on $A$.

Definition 2.16. A many-sorted closure operator $J$ on an $S$-sorted set $A$ is algebraic if, for every $X \subseteq A, J(X)=\bigcup_{K \subseteq_{\text {fin }} X} J(K)$, and is uniform if, for every $X, Y \subseteq A$, if $\operatorname{supp}_{S}(X)=\operatorname{supp}_{S}(Y)$, then $\operatorname{supp}_{S}(J(X))=\operatorname{supp}_{S}(J(Y))$.

We next prove that, for a many-sorted closure operator, the property of being $n$-ary is stronger than that of being algebraic.

Proposition 2.17. Let $n$ be a natural number. If a many-sorted closure operator $J$ on an $S$-sorted set $A$ is n-ary, then $J$ is an algebraic many-sorted closure operator on $A$.

Proof. Let $J$ be an $n$-ary many-sorted closure operator on an $S$-sorted set $A$ and let $X$ be a subset of $A$. Then, obviously, $\bigcup_{K \subseteq_{\text {fin }} X} J(K) \subseteq$ $J(X)$. Since $J(X)=J_{\leq n}^{\omega}(X)=\bigcup_{m \in \mathbb{N}} J_{\leq n}^{m}(X)$, to prove that $J(X) \subseteq$ $\bigcup_{K \subseteq_{\text {fin } X}} J(K)$ it suffices to prove that, for every $m \in \mathbb{N}, J_{\leq n}^{m}(X) \subseteq$ $\bigcup_{K \subseteq_{\text {fin } X}} J(K)$.

For $m=0$, since $J_{\leq n}^{0}(X)=X$, we have that $J_{\leq n}^{0}(X) \subseteq \bigcup_{K \subseteq_{\text {fin }} X} J(K)$.

Let $m$ be $k+1$ with $k \geq 0$ and let us suppose that $J_{\leq n}^{k}(X) \subseteq$ $\bigcup_{K \subseteq_{\text {fin } X}} J(K)$. We want to prove that $J_{\leq n}^{k+1}(X) \subseteq \bigcup_{K \subseteq_{\text {fin }} X} J(K)$. However, by definition, $J_{\leq n}^{k+1}(X)=\bigcup\left\{J(Z) \mid Z \in \operatorname{Sub}_{\leq n}\left(J_{\leq n}^{k}(X)\right)\right\}$. Thus it suffices to prove that, for every $Z \in \operatorname{Sub}_{\leq n}\left(J_{\leq n}^{k}(X)\right), J(Z) \subseteq$ 
$\bigcup_{K \subseteq_{\text {fin } X}} J(K)$. Let $Z$ be a subset of $J_{\leq n}^{k}(X)$ such that $\operatorname{card}(Z) \leq n$. Then, since, by the induction hypothesis, $J_{\leq n}^{k}(X) \subseteq \bigcup_{K \subseteq_{\text {fin }} X} J(K)$, we have that $Z \subseteq \bigcup_{K \subseteq_{\text {fin }} X} J(K)$ and, in addition, that card $(Z) \leq n$. Hence, for some $\ell \in \mathbb{N}, \operatorname{supp}_{S}(Z)=\left\{s_{0}, \ldots, s_{\ell-1}\right\}$ and, for every $\alpha \in \ell$, there exists an $n_{\alpha} \in \mathbb{N}-1$ such that $Z_{s_{\alpha}}=\left\{z_{\alpha, 0}, \ldots, z_{\alpha, n_{\alpha}-1}\right\}$. Therefore, for every $\alpha \in \ell$ and every $\beta \in n_{\alpha}$ there exists a $K^{\alpha, \beta} \subseteq_{\text {fin }} X$ such that that $z_{\alpha, \beta} \in J\left(K^{\alpha, \beta}\right)_{s_{\alpha}}$. Since it may be helpful for the sake of understanding, let us represent the situation just described by the following figure:

$$
\begin{array}{ccc}
z_{0,0} \in J\left(K^{0,0}\right)_{s_{0}} & \cdots & z_{0, n_{0}-1} \in J\left(K^{0, n_{0}-1}\right)_{s_{0}} \\
\vdots & \ddots & \vdots \\
z_{\ell-1,0} \in J\left(K^{\ell-1,0}\right)_{s_{\ell-1}} & \cdots & z_{\ell-1, n_{\ell-1}-1} \in J\left(K^{\ell-1, n_{\ell-1}-1}\right)_{s_{\ell-1}}
\end{array}
$$

Then, for every $\alpha \in \ell, Z_{s_{\alpha}} \subseteq J\left(\bigcup_{\beta \in n_{\alpha}} K^{\alpha, \beta}\right)_{s_{\alpha}}$, where $\bigcup_{\beta \in n_{\alpha}} K^{\alpha, \beta} \subseteq_{\text {fin }}$ $X$. So, for $L=\bigcup_{\alpha \in \ell} \bigcup_{\beta \in n_{\alpha}} K^{\alpha, \beta}$, we have that $L \subseteq_{\text {fin }} X$ and $Z \subseteq J(L)$. Therefore $J(Z) \subseteq J(J(L))=J(L) \subseteq \bigcup_{K \subseteq_{\text {fin } X}} J(K)$.

We next define when a subset $X$ of the underlying $S$-sorted set $A$ of a $\Sigma$-algebra $\mathbf{A}$ is closed under an operation $F_{\sigma}$ of $\mathbf{A}$, as well as when $X$ is a subalgebra of $\mathbf{A}$.

Definition 2.18. Let $\mathbf{A}$ be a $\Sigma$-algebra and $X \subseteq A$. Let $\sigma$ be a formal operation in $\Sigma_{w, s}$. We say that $X$ is closed under the operation $F_{\sigma}: A_{w} \longrightarrow A_{s}$ if, for every $a \in X_{w}, F_{\sigma}(a) \in X_{s}$. We say that $X$ is a subalgebra of $\mathbf{A}$ if $X$ is closed under the operations of $\mathbf{A}$. We denote by $\operatorname{Sub}(\mathbf{A})$ the set of all subalgebras of $\mathbf{A}$ (which is an algebraic closure system on $A$ ).

Definition 2.19. Let $\mathbf{A}$ be a $\Sigma$-algebra. Then we denote by $\mathrm{Sg}_{\mathbf{A}}$ the many-sorted closure operator on $A$ defined as follows:

$$
\operatorname{Sg}_{\mathbf{A}}\left\{\begin{array}{cl}
\operatorname{Sub}(A) & \longrightarrow \operatorname{Sub}(A) \\
X & \longmapsto\{C \in \operatorname{Sub}(\mathbf{A}) \mid X \subseteq C\}
\end{array}\right.
$$

We call $\mathrm{Sg}_{\mathbf{A}}$ the subalgebra generating many-sorted operator on $A$ determined by $\mathbf{A}$. For every $X \subseteq A$, we call $\operatorname{Sg}_{\mathbf{A}}(X)$ the subalgebra of A generated by $X$. Moreover, if $X \subseteq A$ is such that $\operatorname{Sg}_{\mathbf{A}}(X)=A$, then we say that $X$ is an $S$-sorted set of generators of $\mathbf{A}$, or that $X$ generates $\mathbf{A}$. Besides, we say that $\mathbf{A}$ is finitely generated if there exists an $S$-sorted subset $X$ of $A$ such that $X$ generates $\mathbf{A}$ and $\operatorname{card}(X)<\aleph_{0}$.

Proposition 2.20. Let $\mathbf{A}$ be a $\Sigma$-algebra. Then the many-sorted closure operator $\mathrm{Sg}_{\mathbf{A}}$ on $A$ is algebraic, i.e., for every $S$-sorted subset $X$ of $A, \operatorname{Sg}_{\mathbf{A}}(X)=\bigcup_{K \subseteq_{\text {fin }} X} \operatorname{Sg}_{\mathbf{A}}(K)$.

For a $\Sigma$-algebra $\mathbf{A}$ we next provide another, more constructive, description of the algebraic many-sorted closure operator $\mathrm{Sg}_{\mathbf{A}}$, which, in addition, will allow us to state a crucial property of $\mathrm{Sg}_{\mathbf{A}}$. Specifically, that $\mathrm{Sg}_{\mathbf{A}}$ is uniform. 
Definition 2.21. Let $\Sigma$ be an $S$-sorted signature and A a $\Sigma$-algebra.

(1) We denote by $\mathrm{E}_{\mathbf{A}}$ the many-sorted operator on $A$ that assigns to an $S$-sorted subset $X$ of $A, \mathrm{E}_{\mathbf{A}}(X)=X \cup\left(\bigcup_{\sigma \in \Sigma . s} F_{\sigma}\left[X_{\operatorname{ar}(\sigma)}\right]\right)_{s \in S}$, where, for $s \in S, \Sigma_{., s}$ is the set of all many-sorted formal operations $\sigma$ such that the coarity of $\sigma$ is $s$ and for $\operatorname{ar}(\sigma)=w \in S^{\star}$, the arity of $\sigma, X_{\operatorname{ar}(\sigma)}=\prod_{i \in|w|} X_{w_{i}}$.

(2) If $X \subseteq A$, then we define the family $\left(\mathrm{E}_{\mathbf{A}}^{n}(X)\right)_{n \in \mathbb{N}}$ in $\operatorname{Sub}(A)$, recursively, as follows:

$$
\begin{aligned}
\mathrm{E}_{\mathbf{A}}^{0}(X) & =X, \\
\mathrm{E}_{\mathbf{A}}^{n+1}(X) & =\mathrm{E}_{\mathbf{A}}\left(\mathrm{E}_{\mathbf{A}}^{n}(X)\right), n \geq 0 .
\end{aligned}
$$

(3) We denote by $\mathrm{E}_{\mathbf{A}}^{\omega}$ the many-sorted operator on $A$ that assigns to an $S$-sorted subset $X$ of $A, \mathrm{E}_{\mathbf{A}}^{\omega}(X)=\bigcup_{n \in \mathbb{N}} \mathrm{E}_{\mathbf{A}}^{n}(X)$.

Proposition 2.22. Let $\mathbf{A}$ be a $\Sigma$-algebra and $X \subseteq A$, then $\operatorname{Sg}_{\mathbf{A}}(X)=$ $\mathrm{E}_{\mathbf{A}}^{\omega}(X)$.

In [3], on pp. 82, we stated the following proposition (there called Proposition 2.7).

Proposition 2.23. Let $\mathbf{A}$ be a $\Sigma$-algebra and $X, Y \subseteq A$. Then we have that

(1) If $\operatorname{supp}_{S}(X)=\operatorname{supp}_{S}(Y)$, then, for every $n \in \mathbb{N}, \operatorname{supp}_{S}\left(\mathrm{E}_{\mathbf{A}}^{n}(X)\right)=$ $\operatorname{supp}_{S}\left(\mathrm{E}_{\mathbf{A}}^{n}(Y)\right)$.

(2) $\operatorname{supp}_{S}\left(\operatorname{Sg}_{\mathbf{A}}(X)\right)=\bigcup_{n \in \mathbb{N}} \operatorname{supp}_{S}\left(\mathrm{E}_{\mathbf{A}}^{n}(X)\right)$.

(3) If $\operatorname{supp}_{S}(X)=\operatorname{supp}_{S}(Y)$, then $\operatorname{supp}_{S}\left(\operatorname{Sg}_{\mathbf{A}}(X)\right)=\operatorname{supp}_{S}\left(\operatorname{Sg}_{\mathbf{A}}(Y)\right)$.

Therefore the algebraic many-sorted closure operator $\mathrm{Sg}_{\mathbf{A}}$ is uniform.

Proposition 2.24. If $\mathbf{A}$ is a finitely generated $\Sigma$-algebra, then every $S$-sorted set of generators of $\mathbf{A}$ contains a finite $S$-sorted subset which also generates $\mathbf{A}$.

Corollary 2.25. If $\mathbf{A}$ is a finitely generated $\Sigma$-algebra, then we have that $\operatorname{IrB}\left(A, \mathrm{Sg}_{\mathbf{A}}\right)$ is not empty.

\section{A CHARACTERIZATION OF THE $n$-ARY MANY-SORTED ClOSURE OPERATORS.}

A theorem of Birkhoff-Frink (see [1]) asserts that every algebraic closure operator on an ordinary set arises, from some algebraic structure on the set, as the corresponding generated subalgebra operator. However, for many-sorted sets such a theorem is not longer true without qualification. In [3], on pp. 83-84, Theorem 3.1 and Corollary 3.2, we characterized the corresponding many-sorted closure operators as precisely the uniform algebraic operators. We next recall the just mentioned characterization since it will be applied afterwards to provide 
a characterization of the $n$-ary many-sorted closure operators on an $S$-sorted set.

Let us notice that in what follows, for a word $w:|w| \rightarrow S$ on $S$, with $|w|$ the lenght of $w$, and an $s \in S$, we denote by $w^{-1}[s]$ the set $\{i \in|w| \mid w(i)=s\}$, and by $\operatorname{Im}(w)$ the set $\{w(i)|i \in| w \mid\}$

Theorem 3.1. Let $J$ be an algebraic many-sorted closure operator on an $S$-sorted set $A$. If $J$ is uniform, then $J=\mathrm{Sg}_{\mathbf{A}}$ for some $S$-sorted signature $\Sigma$ and some $\Sigma$-algebra $\mathbf{A}$.

Proof. Let $\Sigma=\left(\Sigma_{w, s}\right)_{(w, s) \in S^{\star} \times S}$ be the $S$-sorted signature defined, for every $(w, s) \in S^{\star} \times S$, as follows:

$\Sigma_{w, s}=\left\{(X, b) \in \bigcup_{X \in \operatorname{Sub}(A)}\left(\{X\} \times J(X)_{s}\right) \mid \forall t \in S\left(\operatorname{card}\left(X_{t}\right)=|w|_{t}\right)\right\}$,

where for a sort $s \in S$ and a word $w:|w| \rightarrow S$ on $S$, with $|w|$ the lenght of $w$, the number of occurrences of $s$ in $w$, denoted by $|w|_{s}$, is $\operatorname{card}\left(w^{-1}[s]\right)$.

Before proceeding any further, let us remark that, for $(w, s) \in S^{\star} \times S$ and $(X, b) \in \bigcup_{X \in \operatorname{Sub}(A)}\left(\{X\} \times J(X)_{s}\right)$, the following conditions are equivalent:

(1) $(X, b) \in \Sigma_{w, s}$, i.e., for every $t \in S, \operatorname{card}\left(X_{t}\right)=|w|_{t}$.

(2) $\operatorname{supp}_{S}(X)=\operatorname{Im}(w)$ and, for every $t \in \operatorname{supp}_{S}(X), \operatorname{card}\left(X_{t}\right)=$ $|w|_{t}$.

On the other hand, for the index set $\Lambda=\bigcup_{Y \in \operatorname{Sub}(A)}\left(\{Y\} \times \operatorname{supp}_{S}(Y)\right)$ and the $\Lambda$-indexed family $\left(Y_{s}\right)_{(Y, s) \in \Lambda}$ whose $(Y, s)$-th coordinate is $Y_{s}$, precisely the $s$-th coordinate of the $S$-sorted set $Y$ of the index $(Y, s) \in$ $\Lambda$, let $f$ be a choice function for $\left(Y_{s}\right)_{(Y, s) \in \Lambda}$, i.e., an element of $\prod_{(Y, s) \in \Lambda} Y_{s}$.

Moreover, for every $w \in S^{\star}$ and $a \in \prod_{i \in|w|} A_{w(i)}$, let $M^{w, a}=$ $\left(M_{s}^{w, a}\right)_{s \in S}$ be the finite $S$-sorted subset of $A$ defined as $M_{s}^{w, a}=\left\{a_{i} \mid\right.$ $\left.i \in w^{-1}[s]\right\}$, for every $s \in S$.

Now, for $(w, s) \in S^{\star} \times S$ and $(X, b) \in \Sigma_{w, s}$, let $F_{X, b}$ be the manysorted operation from $\prod_{i \in|w|} A_{w(i)}$ into $A_{s}$ that to an $a \in \prod_{i \in|w|} A_{w(i)}$ assigns $b$, if $M^{w, a}=X$ and $f\left(J\left(M^{w, a}\right), s\right)$, otherwise.

We will prove that the $\Sigma$-algebra $\mathbf{A}=(A, F)$ is such that $J=$ $\mathrm{Sg}_{\mathbf{A}}$. But before doing that it is necessary to verify that the definition of the many-sorted operations is sound, i.e., that for every $(w, s) \in S^{\star} \times S,(X, b) \in \Sigma_{w, s}$ and $a \in \prod_{i \in|w|} A_{w(i)}$, it happens that $s \in$ $\operatorname{supp}_{S}\left(J\left(M^{w, a}\right)\right)$, and for this it suffices to prove that $\operatorname{supp}_{S}\left(M^{w, a}\right)=$ $\operatorname{supp}_{S}(X)$, because, by hypothesis, $J$ is uniform and, by definition, $b \in J(X)_{s}$.

If $t \in \operatorname{supp}_{S}\left(M^{w, a}\right)$, then $M_{t}^{w, a}$ is nonempty, i.e., there exists an $i \in|w|$ such that $w(i)=t$. Therefore, because $(X, b) \in \Sigma_{w, s}$, we have that $0<|w|_{t}=\operatorname{card}\left(X_{t}\right)$, hence $t \in \operatorname{supp}_{S}(X)$.

Reciprocally, if $t \in \operatorname{supp}_{S}(X),|w|_{t}>0$, and there is an $i \in|w|$ such that $w(i)=t$, hence $a_{i} \in A_{t}$, and from this we conclude that $M_{t}^{w, a} \neq \varnothing$, 
i.e., that $t \in \operatorname{supp}_{S}\left(M^{w, a}\right)$. Therefore, $\operatorname{supp}_{S}\left(M^{w, a}\right)=\operatorname{supp}_{S}(X)$ and, by the uniformity of $J, \operatorname{supp}_{S}\left(J\left(M^{w, a}\right)\right)=\operatorname{supp}_{S}(J(X))$. But, by definition, $b \in J(X)_{s}$, so $s \in \operatorname{supp}_{S}\left(J\left(M^{w, a}\right)\right)$ and the definition is sound.

Now we prove that, for every $X \subseteq A, J(X) \subseteq \operatorname{Sg}_{\mathbf{A}}(X)$. Let $X$ be an $S$-sorted subset of $A, s \in S$ and $b \in J(X)_{s}$. Then, because $J$ is algebraic, $b \in J(Y)_{s}$, for some finite $S$-sorted subset $Y$ of $X$. From such an $Y$ we will define a word $w_{Y}$ in $S$ and an element $a_{Y}$ of $\prod_{i \in\left|w_{Y}\right|} A_{w_{Y}(i)}$ such that

(1) $Y=M^{w_{Y}, a_{Y}}$,

(2) $(Y, b) \in \Sigma_{w_{Y}, s}$, i.e., $b \in J(Y)_{s}$ and, for all $t \in S$, $\operatorname{card}\left(Y_{t}\right)=$ $\left|w_{Y}\right|_{t}$, and

(3) $a_{Y} \in \prod_{i \in\left|w_{Y}\right|} X_{w_{Y}(i)}$,

then, because $F_{Y, b}\left(a_{Y}\right)=b$, we will be entitled to assert that $b \in$ $\operatorname{Sg}_{\mathbf{A}}(X)_{s}$.

But given that $Y$ is finite if, and only if, $\operatorname{supp}_{S}(Y)$ is finite and, for every $t \in \operatorname{supp}_{S}(Y), Y_{t}$ is finite, let $\left\{s_{\alpha} \mid \alpha \in m\right\}$ be an enumeration of $\operatorname{supp}_{S}(Y)$ and, for every $\alpha \in m$, let $\left\{y_{\alpha, i} \mid i \in p_{\alpha}\right\}$ be an enumeration of the nonempty $s_{\alpha}$-th coordinate, $Y_{s_{\alpha}}$, of $Y$. Then we define, on the one hand, the word $w_{Y}$ as the mapping from $\left|w_{Y}\right|=\sum_{\alpha \in m} p_{\alpha}$ into $S$ such that, for every $i \in\left|w_{Y}\right|$ and $\alpha \in m, w_{Y}(i)=s_{\alpha}$ if, and only if, $\sum_{\beta \in \alpha} p_{\beta} \leq i \leq \sum_{\beta \in \alpha+1} p_{\beta}-1$ and, on the other hand, the element $a_{Y}$ of $\prod_{i \in\left|w_{Y}\right|} A_{w_{Y}(i)}$ as the mapping from $\left|w_{Y}\right|$ into $\bigcup_{i \in\left|w_{Y}\right|} A_{w_{Y}(i)}$ such that, for every $i \in\left|w_{Y}\right|$ and $\alpha \in m, a_{Y}(i)=y_{\alpha, i-\sum_{\beta \in \alpha} p_{\beta}}$ if, and only if, $\sum_{\beta \in \alpha} p_{\beta} \leq i \leq \sum_{\beta \in \alpha+1} p_{\beta}-1$. From these definitions follow (1), (2) and (3) above. Let us observe that (1) is a particular case of the fact that the mapping $M$ from $\bigcup_{w \in S^{\star}}\left(\{w\} \times \prod_{i \in|w|} A_{w(i)}\right)$ into $\operatorname{Sub}_{\text {fin }}(A)$ that to a pair $(w, a)$ assigns $M^{w, a}$ is surjective.

From the above and the definition of $F_{Y, b}$ we can affirm that $F_{Y, b}\left(a_{Y}\right)=$ $b$, hence $b \in \operatorname{Sg}_{\mathbf{A}}(X)_{s}$. Therefore $J(X) \subseteq \operatorname{Sg}_{\mathbf{A}}(X)$.

Finally, we prove that, for every $X \subseteq A, \operatorname{Sg}_{\mathbf{A}}(X) \subseteq J(X)$. But for this, by Proposition 2.22, it is enough to prove that, for every subset $X$ of $A$, we have that $\mathrm{E}_{\mathbf{A}}(X) \subseteq J(X)$. Let $s \in S$ be and $c \in \mathrm{E}_{\mathbf{A}}(X)_{s}$. If $c \in X_{s}$, then $c \in J(X)_{s}$, because $J$ is extensive. If $c \notin X_{s}$, then, by the definition of $\mathrm{E}_{\mathbf{A}}(X)$, there exists a word $w \in S^{\star}$, a manysorted formal operation $(Y, b) \in \Sigma_{w, s}$ and an $a \in \prod_{i \in|w|} X_{w(i)}$ such that $F_{Y, b}(a)=c$. If $M^{w, a}=Y$, then $c=b$, hence $c \in J(Y)_{s}$, therefore, because $M^{w, a} \subseteq X, c \in J(X)_{s}$. If $M^{w, a} \neq Y$, then $F_{Y, b}(a) \in J\left(M^{w, a}\right)_{s}$, but, because $M^{w, a} \subseteq X$ and $J$ is isotone, $J\left(M^{w, a}\right)$ is a subset of $J(X)$, hence $F_{Y, b}(a) \in J(X)_{s}$. Therefore $\mathrm{E}_{\mathbf{A}}(X) \subseteq J(X)$.

The just stated theorem together with Proposition 2.23 entails the following corollary. 
Corollary 3.2. Let $J$ be an algebraic many-sorted closure operator on an $S$-sorted set $A$. Then $J=\operatorname{Sg}_{\mathbf{A}}$ for some $S$-sorted signature $\Sigma$ and some $\Sigma$-algebra $\mathbf{A}$ if, and only if, $J$ is uniform.

We next prove that for a natural number $n$, an $S$-sorted signature $\Sigma$, and a $\Sigma$-algebra $\mathbf{A}$, under a suitable condition on $\Sigma$ related to $n$, the uniform algebraic many-sorted closure operator $\mathrm{Sg}_{\mathbf{A}}$ is an $n$-ary many-sorted closure operator on $A$.

Proposition 3.3. Let $\Sigma$ be an $S$-sorted signature, A a $\Sigma$-algebra, and $n \in \mathbb{N}$. If $\Sigma$ is such that, for every $(w, s) \in S^{\star} \times S, \Sigma_{w, s}=\varnothing$ if $|w|>n$-in which case we will say that every operation of $\mathbf{A}$ is of an arity $\leq n-$, then the uniform algebraic many-sorted closure operator $\mathrm{Sg}_{\mathbf{A}}$ is an $n$-ary many-sorted closure operator on $A$, i.e., $\mathrm{Sg}_{\mathbf{A}}=\left(\mathrm{Sg}_{\mathbf{A}}\right)_{\leq n}^{\omega}$.

Proof. It follows from $\operatorname{Sg}_{\mathbf{A}}(X)=\mathrm{E}_{\mathbf{A}}^{\omega}(X)$ and from the fact that, for every $X \subseteq A, \mathrm{E}_{\mathbf{A}}(X) \subseteq\left(\operatorname{Sg}_{\mathbf{A}}\right)_{\leq n}(X) \subseteq \operatorname{Sg}_{\mathbf{A}}(X)$. The details are left to the reader. However, we notice that it is advisable to split the proof into two cases, one for $n=0$ and another one for $n \geq 1$.

Proposition 3.4. Let $A$ be an $S$-sorted set, $J$ a many-sorted closure operator on $A$, and $n \in \mathbb{N}$. If $J$ is n-ary (hence, by Proposition 2.17, algebraic) and uniform, then there exists an $S$-sorted signature $\Sigma^{\prime}$ and a $\Sigma^{\prime}$-algebra $\mathbf{A}^{\prime}$ such that $J=\operatorname{Sg}_{\mathbf{A}^{\prime}}$ and every operation of $\mathbf{A}^{\prime}$ is of an arity $\leq n$.

Proof. If we denote by $\mathbf{A}=(A, F)$ the $\Sigma$-algebra associated to $J$ constructed in the proof of Theorem 3.1, then taking as $\Sigma^{\prime}$ the $S$-sorted signature defined, for every $(w, s) \in S^{\star} \times S$, as: $\Sigma_{w, s}^{\prime}=\Sigma_{w, s}$, if $|w| \leq n$; and $\Sigma_{w, s}^{\prime}=\varnothing$, if $|w|>n$, and as $\mathbf{A}^{\prime}=\left(A^{\prime}, F^{\prime}\right)$ the $\Sigma^{\prime}$-algebra defined as: $A^{\prime}=A$, and $F^{\prime}=F \circ$ inc $^{\Sigma^{\prime}, \Sigma}$, where inc ${ }^{\Sigma^{\prime}, \Sigma}=\left(\operatorname{inc}_{w, s}^{\Sigma^{\prime}, \Sigma}\right)_{(w, s) \in S^{\star} \times S}$ is the canonical inclusion of $\Sigma^{\prime}$ into $\Sigma$, then one can show that $J=\operatorname{Sg}_{\mathbf{A}^{\prime}}$.

From the just stated proposition together with Proposition 3.3 it follows immediately the following corollary, which is an algebraic characterization of the $n$-ary and uniform many-sorted closure operators.

Corollary 3.5. Let $J$ be a many-sorted closure operator on an $S$ sorted set $A$ and $n \in \mathbb{N}$. Then $J$ is $n$-ary and uniform if, and only if, there exists an $S$-sorted signature $\Sigma$ and a $\Sigma$-algebra A such that $J=\mathrm{Sg}_{\mathbf{A}}$ and every operation of $\mathbf{A}$ is of an arity $\leq n$.

\section{THE IRREDUNDANT BASIS THEOREM FOR MANY-SORTED CLOSURE SPACES.}

We next show Tarski's irredundant basis theorem for many-sorted closure spaces.

Theorem 4.1 (Tarski's irredundant basis theorem for many-sorted closure spaces). Let $(A, J)$ be a many-sorted closure space. If $J$ is an 
$n$-ary many-sorted operator on the $S$-sorted set $A$, with $n \geq 2$, and if $i<j$ with $i, j \in \operatorname{IrB}_{J}(A)$ such that

$$
\{i+1, \ldots, j-1\} \cap \operatorname{IrB}_{J}(A)=\varnothing,
$$

then $j-i \leq n-1$. In particular, if $n=2$, then $\operatorname{IrB}_{J}(A)$ is a convex subset of $\mathbb{N}$.

Proof. Let $Z \subseteq A$ be an irredundant basis with respect to $J$ such that $\operatorname{card}(Z)=j$ and $\mathcal{K}=\left\{X \in \operatorname{IrB}_{J}(A) \mid \operatorname{card}(X) \leq i\right\}$. Since $J$ is $n$-ary, we can assert that $J(Z)=A=\bigcup_{m \in \mathbb{N}} J_{\leq n}^{m}(Z)$, so, for every $s \in S$, $J(Z)_{s}=A_{s}=\bigcup_{m \in \mathbb{N}} J_{\leq n}^{m}(Z)_{s}$. Let $X$ be an element of $\mathcal{K}$. Then there exists a $k \in \mathbb{N}-1$ such that $X \subseteq J_{\leq n}^{k}(Z)$. The natural number $k$ should be strictly greater than 0 , because if $k=0, X \subseteq J_{\leq n}^{0}(Z)=Z$, but $\operatorname{card}(X)=i<j=\operatorname{card}(Z)$, so $Z$ would not be an irredundant basis. So that, for every $X \in \mathcal{K},\left\{k \in \mathbb{N}-1 \mid X \subseteq J_{\leq n}^{k}(Z)\right\} \neq \varnothing$. Therefore, for every $X \in \mathcal{K}$, we can choose the least element of such a set, denoted by $d_{Z}(X)$, and there is fulfilled that $d_{Z}(X)$ is greater than or equal to 1 . For $d_{Z}(X)-1$ we have that $X \nsubseteq J_{\leq n}^{d_{Z}(X)-1}(Z)$. So we conclude that there exists a mapping $d_{Z}: \mathcal{K} \longrightarrow \mathbb{N}-1$ that to an $X \in \mathcal{K}$ assigns $d_{Z}(X)$. The image of the mapping $d_{Z}$, which is a nonempty part of $\mathbb{N}-1$, is well-ordered, hence it has a least element, which is, necessarily, non zero, $t+1$, therefore, since $\mathcal{K} / \operatorname{Ker}\left(d_{Z}\right)$ is isomorphic to $\operatorname{Im}\left(d_{Z}\right)$, by transport of structure, it will also be wellordered, then we can always choose an $X \in \mathcal{K}$ such that, for every $Y \in \mathcal{K}, d_{Z}(X) \leq d_{Z}(Y)$, e.g., an $X$ such that its equivalence class corresponds to the minimum $t+1$ of $\operatorname{Im}\left(d_{Z}\right)$. Moreover, among the $X$ which have the just mentioned property, we choose an $X^{0}$ such that, for every $Y \in \mathcal{K}$ with $Y \subseteq J_{\leq n}^{t+1}(Z)$, it happens that

$$
\operatorname{card}\left(X^{0} \cap\left(J_{\leq n}^{t+1}(Z)-J_{\leq n}^{t}(Z)\right)\right) \leq \operatorname{card}\left(Y \cap\left(J_{\leq n}^{t+1}(Z)-J_{\leq n}^{t}(Z)\right)\right) .
$$

By the method of election we have that $X^{0} \subseteq J_{\leq n}^{t+1}(Z)$ but $X^{0} \nsubseteq$ $J_{\leq n}^{t}(Z)$. Of the latter we conclude that there exists an $s_{0} \in S$ such that $X_{s_{0}}^{0} \nsubseteq J_{\leq n}^{t}(Z)_{s_{0}}$, therefore

$$
\left(J_{\leq n}^{t+1}(Z)_{s_{0}}-J_{\leq n}^{t}(Z)_{s_{0}}\right) \cap X_{s_{0}}^{0} \neq \varnothing .
$$

Let $a_{0} \in\left(J_{\leq n}^{t+1}(Z)_{s_{0}}-J_{\leq n}^{t}(Z)_{s_{0}}\right) \cap X_{s_{0}}^{0}$ be. Then $a_{0} \in X_{s_{0}}^{0}, a_{0} \in$ $J_{\leq n}^{t+1}(Z)_{s_{0}}$ but $a_{0} \notin J_{\leq n}^{t}(Z)_{s_{0}}$. However, $J_{\leq n}^{t+1}(Z)=J_{\leq n}\left(J_{\leq n}^{t}(Z)\right)$, by definition, hence there exists a part $F$ of $J_{\leq n}^{t}(Z)$ such that card $(F) \leq n$ and $a_{0} \in J(F)_{s_{0}}$. Let $X^{1}$ be the part of $A$ defined as follows:

$$
X_{s}^{1}= \begin{cases}X_{s}^{0} \cup F_{s}, & \text { if } s \neq s_{0} \\ \left(X_{s_{0}}^{0}-\left\{a_{0}\right\}\right) \cup F_{s_{0}}, & \text { if } s=s_{0} .\end{cases}
$$

It holds that $X^{0} \subseteq J\left(X^{1}\right)$. Therefore $J\left(X^{0}\right) \subseteq J\left(X^{1}\right)$, but $J\left(X^{0}\right)=$ $A$, hence $J\left(X^{1}\right)=A$, i.e., $X^{1}$ is a finite generator with respect to $J$, thus $X^{1}$ will contain a minimal generator $X^{2}$ with respect to $J$. It 
holds that $\operatorname{card}\left(X^{2}\right) \leq \operatorname{card}\left(X^{1}\right)<\operatorname{card}\left(X^{0}\right)+n$. It cannot happen that $\operatorname{card}\left(X^{0}\right)+n \leq j$. Because if $\operatorname{card}\left(X^{0}\right)+n \leq j$, then $\operatorname{card}\left(X^{2}\right)<j$, hence, since

$$
\{i+1, \ldots, j-1\} \cap \operatorname{IrB}(A, J)=\varnothing,
$$

$X^{2} \in \mathcal{K}$, but $X^{2} \subseteq J_{\leq n}^{t+1}(Z)$ and, moreover, it happens that

$$
\operatorname{card}\left(X^{2} \cap\left(J_{\leq n}^{t+1}(Z)-J_{\leq n}^{t}(Z)\right)\right)<\operatorname{card}\left(X^{0} \cap\left(J_{\leq n}^{t+1}(Z)-J_{\leq n}^{t}(Z)\right)\right),
$$

because $a_{0} \notin X_{s_{0}}^{2}$ but $a_{0} \in X_{s_{0}}^{0}$, which contradicts the choice of $X^{0}$. Hence $\operatorname{card}\left(X^{0}\right)+n>j$. But $\operatorname{card}\left(X^{0}\right) \leq i$, therefore $j-i<n$, i.e., $j-i \leq n-1$.

\section{REFERENCES}

[1] G. Birkhoff and O. Frink, Representation of lattices by sets. Trans. Amer. Math. Soc., 64 (1948), pp. 299-316.

[2] S. Burris and H.P. Sankappanavar, A course in universal algebra, SpringerVelag, 1981.

[3] J. Climent Vidal and J. Soliveres Tur, On many-sorted algebraic closure operators. Math. Nachr. 266 (2004), pp. 81-84.

[4] A. Tarski, An interpolation theorem for irredundant bases of closure operators, Discrete Math. 12 (1975), pp. 185-192.

Universitat de València, Departament de Lògica i Filosofia de la CiÈncia, Av. Blasco IbÁÑez, 30-7ª , 46010 ValènCia, Spain

E-mail address: Juan.B.Climent@uv.es

Universitat de València, Departament d’Àlgebra, Dr. Moliner, 50, 46100 BurJassot, VALÈnCIA, SpAin

E-mail address: Enric.Cosme@uv.es 